\title{
Theory of the Pseudospin Resonance in Semiconductor Bilayers
}

\author{
Saeed H. Abedinpour, ${ }^{1}$ Marco Polini, ${ }^{1, *}$ A. H. MacDonald, ${ }^{2}$ B. Tanatar, ${ }^{3}$ M. P. Tosi,${ }^{1}$ and G. Vignale ${ }^{4}$ \\ ${ }^{1}$ NEST-CNR-INFM and Scuola Normale Superiore, I-56126 Pisa, Italy \\ ${ }^{2}$ Department of Physics, The University of Texas at Austin, Austin, Texas 78712, USA \\ ${ }^{3}$ Department of Physics, Bilkent University, Bilkent, 06800 Ankara, Turkey \\ ${ }^{4}$ Department of Physics and Astronomy, University of Missouri-Columbia, Columbia, Missouri 65211, USA
}

(Received 12 June 2007; published 14 November 2007)

\begin{abstract}
The pseudospin degree of freedom in a semiconductor bilayer gives rise to a collective mode analogous to the ferromagnetic-resonance mode of a ferromagnet. We present a many-body theory of the dependence of the energy and the damping of this mode on layer separation $d$. Based on these results, we discuss the possibilities of realizing transport-current driven pseudospin-transfer oscillators in semiconductors, and of using the pseudospin-transfer effect as an experimental probe of intersubband plasmons.
\end{abstract}

PACS numbers: 73.21.-b, 71.10.Ca, 76.50.+g, 85.75.-d

Introduction. - The layer degree of freedom in semiconductor bilayers is often regarded [1] as an effective spin- $1 / 2$ pseudospin degree of freedom in which electrons in the top layer are assigned one pseudospin state, and electrons in the other layer the opposite one. In the quantum Hall regime [2] (high magnetic field), or, possibly, at zero magnetic field but extremely low densities [3], electron bilayers are sometimes pseudospin ferromagnets. The appearance of these broken-symmetry states has motivated a long-standing interest in phenomena which are pseudospin analogs of the very robust magnetoelectric effects which underpin spintronics in ferromagnetic metals. Although spontaneous pseudospin polarization does not usually occur at zero field, tunneling between the two layers acts as an effective magnetic field which leads to a finite pseudospin magnetization and to a pseudospin resonance analogous to the ferromagnetic resonance of conventional magnetized materials. This resonance-better known in the semiconductor literature as the transverse or intersubband plasmon [4] - is of fundamental interest because both its frequency and its linewidth depend sensitively on many-body effects which cannot be completely described in the random phase approximation (RPA) [5].

This Letter develops the theory of the pseudospin resonance in three ways. First of all, we apply a new theoretical approach which is distinctly superior to the RPA [6] and its extensions [7], becoming exact in the limit in which the difference $V_{-}$between interlayer and intralayer electron-electron interaction is small. In particular, the calculation of the linewidth to second order in $V_{-}$is exact and equivalent to the calculation of the Gilbert damping $[8,9]$ in real spin dynamics. Second, we point out the feasibility of a new semiconductor device, which is analogous to the spin-transfer oscillator [10] of ordinary spintronics. In a spin-transfer oscillator, spin-polarized currents drive ferromagnetic-resonance collective spindynamics in the presence of applied fields strong enough to oppose hysteretic switching. In a semiconductor bilayer pseudospin-polarized currents (corresponding to an inter- layer tunneling current, easily realizable using individuallayer contacting techniques [11]) could, provided that the resonance is sufficiently sharp, drive collective pseudospin dynamics and yield a device with similar functionality. Finally, we point out that the possibility of driving transverse plasmons by means of a tunneling current opens a new avenue for experimental studies of these modes, which have so far been studied only by inelastic light scattering.

The model. - In a bilayer, electrons in the same layer interact through the two-dimensional (2D) Coulomb interaction $V_{s}(q)=2 \pi e^{2} /(\epsilon q)$ ( $\epsilon$ is the dielectric constant), while electrons in different layers are coupled through the interlayer Coulomb interaction $V_{d}(q)=V_{s}(q) e^{-q d}$. The fact that $V_{s} \neq V_{d}$ is ultimately responsible for a nonzero intrinsic linewidth of the pseudospin resonance. We assume a spatially constant interlayer tunneling amplitude which we denote by $\Delta_{\text {SAS }} / 2$ and present our theory using a pseudospin representation in which the tunneling term is diagonal, i.e., the representation in which $|\uparrow\rangle$ refers to the symmetric combination of single-layer states and $|\downarrow\rangle$ to the antisymmetric combination. The total Hamiltonian is then $(\hbar=1)$

$$
\begin{aligned}
\hat{\mathcal{H}}= & -\Delta_{\mathrm{SAS}} \hat{S}_{\mathrm{tot}}^{z}+\sum_{\mathbf{k}, \alpha, \sigma} \frac{\mathbf{k}^{2}}{2 m} \hat{c}_{\mathbf{k}, \alpha, \sigma}^{\dagger} \hat{c}_{\mathbf{k}, \alpha, \sigma} \\
& +\frac{1}{2 S} \sum_{\mathbf{q} \neq 0} V_{+}(q) \hat{\rho}_{\mathbf{q}} \hat{\rho}_{-\mathbf{q}}+\frac{2}{S} \sum_{\mathbf{q} \neq 0} V_{-}(q) \hat{S}_{\mathbf{q}}^{x} \hat{S}_{-\mathbf{q}}^{x} .
\end{aligned}
$$

Here $\sigma$ is the real-spin label, $\alpha$ is the pseudospin label, $S$ is the sample area, $\hat{\rho}_{\mathbf{q}}=\sum_{\mathbf{k}, \alpha, \sigma} \hat{c}_{\mathbf{k}-\mathbf{q} / 2, \alpha, \sigma}^{\dagger} \hat{c}_{\mathbf{k}+\mathbf{q} / 2, \alpha, \sigma}$ and $\hat{S}_{\mathbf{q}}^{a}=\sum_{\mathbf{k}, \alpha, \beta, \sigma} \hat{c}_{\mathbf{k}-\mathbf{q} / 2, \alpha, \sigma}^{\dagger}\left(\tau_{\alpha \beta}^{a} / 2\right) \hat{c}_{\mathbf{k}+\mathbf{q} / 2, \beta, \sigma}$ are the total density and the pseudospin operators ( $\tau^{a}$ being Pauli matrices with $a=x, y, z), \hat{S}_{\text {tot }}^{a}=\hat{S}_{\mathbf{q}=0}^{a}$, and, finally, $V_{ \pm}(q)=$ $\left[V_{s}(q) \pm V_{d}(q)\right] / 2$.

The interlayer tunneling term $\propto \Delta_{\mathrm{SAS}}$ acts as a pseudospin-magnetic field in the $\hat{z}$ direction. The pseudospin resonance then involves collective precession around this pseudospin field, with $\hat{y}$-direction pseudospins repre- 
senting current flowing between the layers and $\hat{x}$-direction pseudospins representing charge accumulation in one of the layers. Note also that, in the representation chosen above, the last term in Eq. (1) breaks rotational invariance around the $\hat{z}$ axis in pseudospin space.

Theory.-The theory we develop in this Letter is based on the observation that the difference between the intra and the interlayer interaction $V_{-}(q)=\pi e^{2}\left(1-e^{-q d}\right) /(\epsilon q)$ is always smaller than $\pi e^{2} d / \epsilon$, which becomes a small perturbation when $d \ll \max \left(r_{s} a_{B}, a_{B} / r_{s}^{2}\right)$. Here $r_{s}=$ $\left(\pi n a_{B}^{2}\right)^{-1 / 2}$ is the Wigner-Seitz density parameter and $a_{B}=\epsilon /\left(m e^{2}\right)$ is the Bohr radius. The above inequality guarantees that the last term in Eq. (1) is a small perturbation either compared to the kinetic energy $\left[\sim e^{2} /\left(r_{s}^{2} \epsilon a_{B}\right)\right]$ which dominates in the high-density limit, or compared to the interaction energy $\left[\sim e^{2} /\left(r_{s} \epsilon a_{B}\right)\right]$ which dominates in the low-density limit. We will, therefore, perform a systematic expansion for the pseudospin resonance frequency and damping rate in powers of $V_{-}(q)$. Our approach will be asymptotically exact in the limit $d \ll a_{B}$ and is expected to be qualitatively correct for $d \sim a_{B}$.

We determine the properties of the pseudospin resonance by evaluating the transverse pseudospin response function $\chi_{S^{x} S^{x}}(q, \omega)=\left\langle\left\langle\hat{S}_{\mathbf{q}}^{x} ; \hat{S}_{-\mathbf{q}}^{x}\right\rangle\right\rangle_{\omega} / S$, where we have introduced the Kubo product $\langle\langle\hat{A} ; \hat{B}\rangle\rangle_{\omega}=-i \lim _{\epsilon \rightarrow 0^{+}} \times$ $\int_{0}^{+\infty} d t e^{i \omega t} e^{-\epsilon t}\left\langle\Psi_{\mathrm{GS}}|[\hat{A}(t), \hat{B}(0)]| \Psi_{\mathrm{GS}}\right\rangle[12],\left|\Psi_{\mathrm{GS}}\right\rangle$ being the ground state. Since our aim is to calculate the transverse mode at $q=0$ we will focus on the response function $\chi_{S^{x} S^{x}}(\omega) \equiv \chi_{S^{x} S^{x}}(q=0, \omega)$. The in-plane pseudospin operators satisfy the Heisenberg equations of motion,

$$
\left\{\begin{array}{l}
\partial_{t} \hat{S}_{\mathrm{tot}}^{x}=\Delta_{\mathrm{SAS}} \hat{S}_{\mathrm{tot}}^{y} \\
\partial_{t} \hat{S}_{\mathrm{tot}}^{y}=-\Delta_{\mathrm{SAS}} \hat{S}_{\mathrm{tot}}^{x}-\frac{2}{S} \sum_{\mathbf{k}} V_{-}(k)\left(\hat{S}_{\mathbf{k}}^{z} \hat{S}_{-\mathbf{k}}^{x}+\hat{S}_{\mathbf{k}}^{x} \hat{S}_{-\mathbf{k}}^{z}\right) ;
\end{array}\right.
$$

$\hat{S}_{\text {tot }}^{x}$, which measures the difference between charges in the two layers, is a good quantum number when $\Delta_{\mathrm{SAS}} \rightarrow 0$, whereas $\hat{S}_{\text {tot }}^{y}$ is not conserved even in this limit because of the pseudospin-dependent interactions. When $d \rightarrow 0$ these equations reduce to a pseudospin version of Larmor's theorem, in which the precession is undamped and its frequency is given exactly by the noninteracting particle value $\Delta_{\text {SAS }}$.

Our theory starts by making repeated use of Eqs. (2) in the Kubo product identity [12,13]: $\langle\langle\hat{A} ; \hat{B}\rangle\rangle_{\omega}=$ $\left\langle\Psi_{\mathrm{GS}}|[\hat{A}, \hat{B}]| \Psi_{\mathrm{GS}}\right\rangle / \omega+i\left\langle\left\langle\partial_{t} \hat{A} ; \hat{B}\right\rangle\right\rangle_{\omega} / \omega$. After some algebraic manipulations we arrive at the following exact expression for $\chi_{S^{x} S^{x}}(\omega)$ :

$$
\begin{aligned}
\chi_{S^{x} S^{x}}(\omega)= & \mathcal{M}^{z} \frac{\Delta_{\mathrm{SAS}}}{\Omega^{2}}+\frac{4 \Delta_{\text {SAS }}^{2}}{\Omega^{4} S^{2}} \sum_{\mathbf{k}} V_{-}(k) f(\mathbf{k}) \\
& +\frac{2 i \omega \Delta_{\mathrm{SAS}}}{\Omega^{4} S^{2}} \sum_{\mathbf{k}} V_{-}(k) g(\mathbf{k}) \\
& +\frac{4 \Delta_{\text {SAS }}^{2}}{\Omega^{4} S^{3}} \sum_{\mathbf{k}, \mathbf{k}^{\prime}} V_{-}(k) V_{-}\left(k^{\prime}\right) \mathcal{L}\left(\mathbf{k}, \mathbf{k}^{\prime}, \omega\right) .
\end{aligned}
$$

Here $\Omega^{2}=\omega^{2}-\Delta_{\text {SAS }}^{2}, \quad \mathcal{M}^{z}=\left\langle\Psi_{\mathrm{GS}}\left|\hat{S}_{\mathrm{tot}}^{z}\right| \Psi_{\mathrm{GS}}\right\rangle / S$ is the ground-state pseudospin magnetization per unit area, $\quad f(\mathbf{k})=\left\langle\Psi_{\mathrm{GS}}\left|\hat{S}_{\mathbf{k}}^{z} \hat{S}_{-\mathbf{k}}^{z}\right| \Psi_{\mathrm{GS}}\right\rangle-\left\langle\Psi_{\mathrm{GS}}\left|\hat{S}_{\mathbf{k}}^{x} \hat{S}_{-\mathbf{k}}^{x}\right| \Psi_{\mathrm{GS}}\right\rangle$, $g(\mathbf{k})=\left\langle\Psi_{\mathrm{GS}}\left|\hat{S}_{\mathbf{k}}^{x} \hat{S}_{-\mathbf{k}}^{y}\right| \Psi_{\mathrm{GS}}\right\rangle+\left\langle\Psi_{\mathrm{GS}}\left|\hat{S}_{\mathbf{k}}^{y} \hat{S}_{-\mathbf{k}}^{x}\right| \Psi_{\mathrm{GS}}\right\rangle, \quad$ and $\mathcal{L}\left(\mathbf{k}, \mathbf{k}^{\prime}, \omega\right)=\left\langle\left\langle\left[\hat{S}_{\mathbf{k}}^{z} \hat{S}_{-\mathbf{k}}^{x}+\hat{S}_{\mathbf{k}}^{x} \hat{S}_{-\mathbf{k}}^{z}\right] ;\left[\hat{S}_{\mathbf{k}^{\prime}}^{z} \hat{S}_{-\mathbf{k}^{\prime}}^{x}+\hat{S}_{\mathbf{k}^{\prime}}^{x} \hat{S}_{-\mathbf{k}^{\prime}}^{z}\right]\right\rangle\right\rangle_{\omega}$. When $V_{-}$is set to zero $(d \rightarrow 0)$, the interaction part of the Hamiltonian is pseudospin invariant. Larmor's theorem then applies to the pseudospin degree of freedom and only the first term on the right-hand side of Eq. (3) survives. We refer to the Hamiltonian $\hat{\mathcal{H}}$ at $V_{-}=0$ as the reference system (RS), on which the perturbative scheme outlined below is based.

The key idea now is to expand $\chi_{S^{x} S^{x}}(\omega)$ in powers of $V_{-}$. For example, the ground-state pseudospin magnetization $\mathcal{M}^{z}$ is expanded as $\mathcal{M}^{z}=\mathcal{M}_{0}^{z}+\mathcal{M}_{1}^{z}+\mathcal{M}_{2}^{z}+\ldots$, where the $n$th term $\mathcal{M}_{n}^{z}$ is $\mathcal{O}\left(V_{-}^{n}\right)$. The quantities $f, g$, and $\mathcal{L}$ are similarly expanded. Note that the zeroth order of $f(\mathbf{k})$, denoted by $f_{0}(\mathbf{k})$, is a nonzero difference between longitudinal and transverse pseudospin structure factors. On the other hand, the zeroth order of $g(\mathbf{k})$ vanishes because the RS Hamiltonian is invariant under rotations by 90 degrees about the $\hat{z}$ axis in pseudospin space which $\operatorname{map} \hat{S}_{x} \rightarrow \hat{S}_{y}$ and $\hat{S}_{y} \rightarrow-\hat{S}_{x}$.

The pseudospin resonance frequency is the solution of the equation $\operatorname{Re}\left[\chi_{S^{x} S^{x}}^{-1}\left(\omega_{\perp}\right)\right]=0$. To appreciate the power of Eq. (3) we first use it to find $\omega_{\perp}$ to first order in $\bar{d}=$ $d / a_{B}$. After some straightforward algebraic manipulations we find that

$$
\omega_{\perp}^{2}=\Delta_{\mathrm{SAS}}^{2}+\frac{4 \Delta_{\mathrm{SAS}} V_{-}(0)}{\mathcal{M}_{0}^{z}} \frac{1}{S^{2}} \sum_{\mathbf{k}} f_{0}(\mathbf{k})+\mathcal{O}\left(\bar{d}^{2}\right)
$$

This equation is exact to all orders in the intralayer Coulomb interaction $V_{s}$. In the high-density limit one can find simple analytical expressions for $\mathcal{M}_{0}^{z}$ and $f_{0}(\mathbf{k})$, $\mathcal{M}_{0}^{z}=\left(n_{\mathrm{S}}-n_{\mathrm{AS}}\right) / 2$ and $S^{-2} \sum_{\mathbf{k}} f_{0}(\mathbf{k})=\left(\mathcal{M}_{0}^{z}\right)^{2} / 2$. Here $n_{\alpha}=k_{F \alpha}^{2} /(2 \pi)$ are the band occupation factors, $k_{F \alpha}$ being the Fermi wave number for band $\alpha$. In this limit Eq. (4) simplifies to $\omega_{\perp}^{2}=\Delta_{\text {SAS }}^{2}+2 \Delta_{\text {SAS }} \mathcal{M}_{0}^{z} V_{-}(0)$. The second term, which supplies the interaction induced shift in the pseudospin resonance position, is a factor of 2 smaller than in RPA theory [6]. The source of this difference is easy to understand: our calculation includes the first-order exchange corrections to the resonance frequency which are absent in the RPA. Since $V_{-}$is independent of $q$ at first order in $d$, corresponding to a $\delta$-function interaction in real space, the like-real-spin contribution to the resonance position shift present in the RPA is canceled by exchange interactions.

The main object of this work is to estimate the resonance decay rate, which appears first at second-order in $V_{-}$and is zero in the RPA (additional interaction corrections to the resonance position $\operatorname{Re}\left(\omega_{\perp}\right)$ also appear at second order [14]). The linewidth of the pseudospin resonance $\left[=-2 \operatorname{Im}\left(\omega_{\perp}\right)\right]$ is given, up to second order in $d$, by 


$$
\Gamma_{\perp}=-\frac{4 V_{-}^{2}(0) \Delta_{\mathrm{SAS}}}{\mathcal{M}_{0}^{z}} \lim _{\omega \rightarrow \Delta_{\mathrm{SAS}}} \frac{\operatorname{Im} \ell_{0}(\omega)}{\omega},
$$

where $\ell_{0}(\omega)$ is the wave-vector sum of the four-spin correlation function $\mathcal{L}_{0}\left(\mathbf{k}, \mathbf{k}^{\prime}, \omega\right)$. This quantity can be eval- uated analytically in the high-density limit in which it is dominated by a decay process where two particle-hole pairs are excited out of the Fermi sea, one involving a pseudospin flip. The second particle-hole excitation is diagonal in pseudospin and absorbs the momentum emitted by the first. We find that

$$
\operatorname{Im} \ell_{0}(\omega)=-\frac{\pi}{2 S^{3}} \sum_{\mathbf{k}, \mathbf{k}^{\prime}, \mathbf{k}^{\prime \prime}} \sum_{\alpha, \beta} \delta\left(\omega-\Omega_{\alpha}\left(\mathbf{k}, \mathbf{k}^{\prime}\right)\right) n_{\mathbf{k}^{\prime \prime}, \alpha} n_{\mathbf{k}^{\prime \prime}-\mathbf{k}+\mathbf{k}^{\prime}, \beta}\left(1-n_{\mathbf{k}^{\prime \prime}+\mathbf{k}^{\prime}, \beta}\right)\left(1-n_{\mathbf{k}^{\prime \prime}-\mathbf{k}, \bar{\alpha}}\right),
$$

where $n_{\mathbf{k}, \alpha}=\Theta\left(k_{F \alpha}-|\mathbf{k}|\right)$ and $\Omega_{\alpha}\left(\mathbf{k}, \mathbf{k}^{\prime}\right)=\mathbf{k} \cdot \mathbf{k}^{\prime} / m+$ $\alpha \Delta_{\text {SAS }}$ [15]. In Fig. 1 we illustrate the dependence of $\operatorname{Im} \ell_{0}(\omega)$ on $\omega$. The $\omega^{3}$ dependence at small $\omega$ is the double-particle-hole excitation manifestation of the familiar Pauli-blocking reduction in the excitation density of states in a Fermi sea which underlies Fermi liquid theory; damping drops much more rapidly at low energies than for ferromagnetic resonance [9] dominated by single-particle decay processes. Equations (3)-(6) constitute the most important results of this work and provide, to the best of our knowledge, the first microscopic theory of the pseudospin resonance linewidth.

Numerical results and discussion. - Typical numerical results for $\Gamma_{\perp}$, calculated from Eqs. (5) and (6), are shown in Figs. 2 and 3. In Fig. 2 we show $\Gamma_{\perp}$ as a function of $\Delta_{\mathrm{SAS}}$ for a bilayer with density $n=8.3 \times 10^{10} \mathrm{~cm}^{-2}$ and interlayer distance $d=L+w=60 \AA$. Here $L=40 \AA$ is the width of each quantum well and $w=20 \AA$ is the barrier width (we have chosen material parameters corresponding to a GaAs/AlGaAs bilayer). The nonmonotonic behavior of $\Gamma_{\perp}$ is entirely due to the crossover in the behavior of $\ell_{0}$ as a function of $\omega$ from the low frequency regime, where $\operatorname{Im} \ell_{0}(\omega) \propto \omega^{3}$, to the large frequency regime where $\operatorname{Im} \ell_{0}(\omega) \rightarrow$ const (see Fig. 1). The nonanalytic behavior

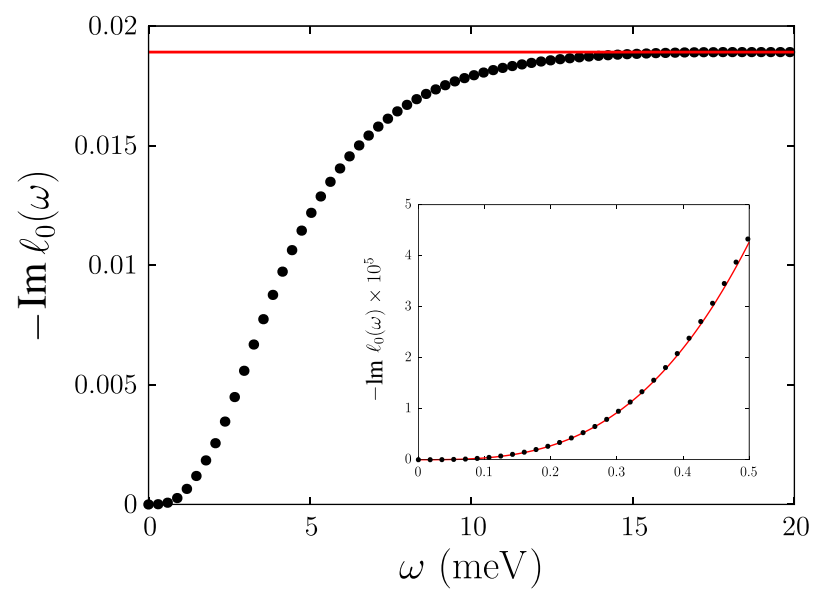

FIG. 1 (color online). Imaginary part of the dynamical response function $\ell_{0}(\omega)$ (in units of $\mathrm{eV}^{-1} \mathrm{~nm}^{-6}$ ) as a function of $\omega$ for a bilayer electron gas with $n=8.3 \times 10^{10} \mathrm{~cm}^{-2}$ and $\Delta_{\mathrm{SAS}}=1.48 \mathrm{meV}$. The solid (red) line is the asymptotic result $\operatorname{Im} \ell_{0}(\omega \rightarrow \infty)=-m n^{2} / 32$. Inset: a zoom of the low-energy region. The solid (red) curve is the expression $\operatorname{Im} \ell_{0}(\omega)=-\gamma \omega^{3}$ with $\gamma \simeq 3.41 \times 10^{-4}[16]$. of $\Gamma_{\perp}$ for $\Delta_{\mathrm{SAS}} \sim 3 \mathrm{meV}$ is due to the transition from the situation in which both symmetric and antisymmetric bands are occupied to that in which only the symmetric band is occupied. In Fig. 3 we illustrate the dependence of $\Gamma_{\perp}$ on density for a fixed value of $\Delta_{\mathrm{SAS}}=1.48 \mathrm{meV}$. Since the resonance frequency is close to $\Delta_{\mathrm{SAS}}$, these calculations predict that the pseudospin resonance can be very sharp, especially when $\Delta_{\mathrm{SAS}}$ is small compared to the Fermi energy of the bilayer. On physical grounds we expect that the main effect of going to higher order in $d$ will be to replace the bare interlayer interaction in Eq. (5) by a weaker screened interaction, further reducing the damping. Note also that the intrinsic linewidth in Eq. (5) provides a lower bound for the actual linewidth: disorder, for example, will tend to increase the linewidth, but this is an extrinsic effect that can, in principle, be reduced by improving the sample quality.

Our theory of the resonance amounts to the derivation of an anisotropic, linearized pseudospin Landau-LifshitzSlonczewski equation:

$$
\left\{\begin{array}{l}
\partial_{t} \mathcal{M}^{x}=\Delta_{\mathrm{SAS}} \mathcal{M}^{y}-\frac{I}{e} \\
\partial_{t} \mathcal{M}^{y}=-\frac{\omega_{\perp}^{2}+\Gamma_{\perp}^{2}}{\Delta_{\mathrm{SAS}}} \mathcal{M}^{x}-\frac{2 \Gamma_{\perp}}{\Delta_{\mathrm{SAS}}} \partial_{t} \mathcal{M}^{x},
\end{array}\right.
$$

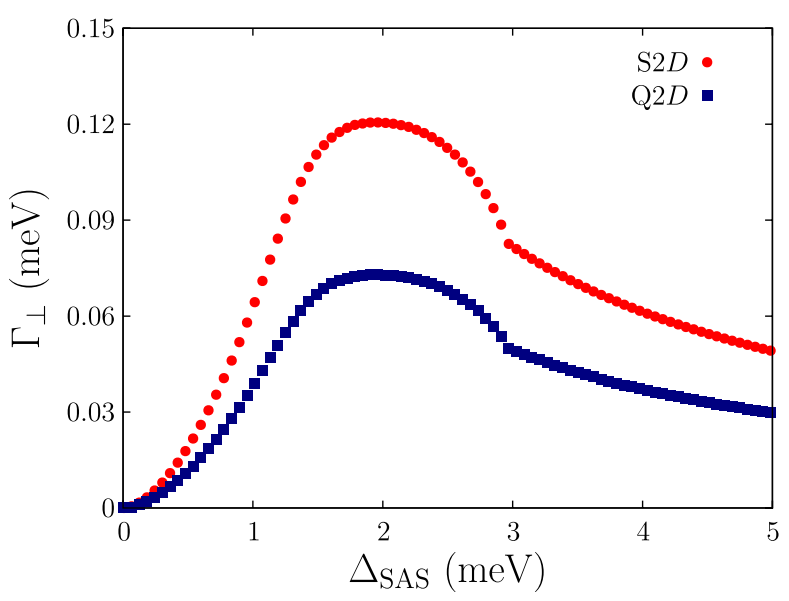

FIG. 2 (color online). Intrinsic linewidth $\Gamma_{\perp}$ of the pseudospin resonance as a function of $\Delta_{\text {SAS }}$ for a bilayer with density $n=$ $8.3 \times 10^{10} \mathrm{~cm}^{-2}$ and $d=60 \AA$. The S2D curve was evaluated using the bare $2 \mathrm{D}$ interactions $V_{s}(q)$ and $V_{d}(q)$ defined above, whereas the Q2D result was evaluated with more realistic interactions weakened by form factors [17] which account for typical quantum well widths. 


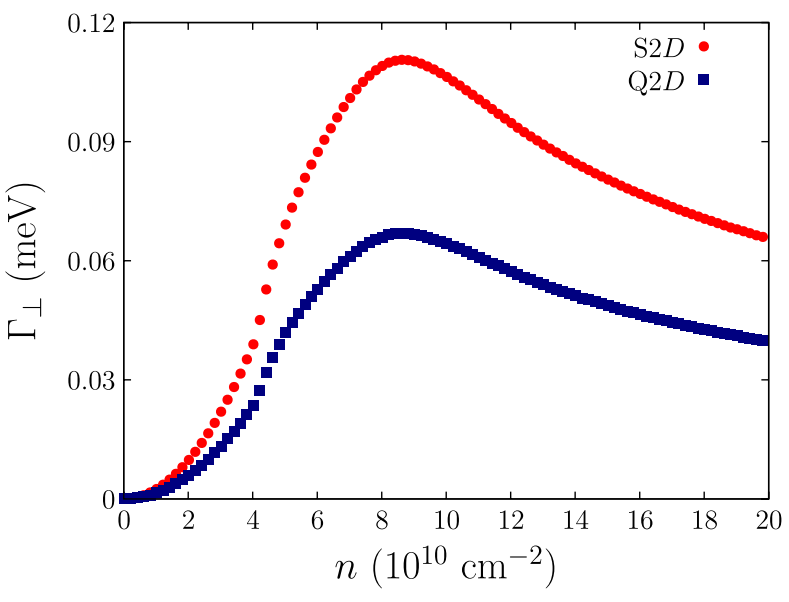

FIG. 3 (color online). Intrinsic linewidth $\Gamma_{\perp}$ of the pseudospin resonance as a function of $n$ for a bilayer with tunneling gap $\Delta_{\mathrm{SAS}}=1.48 \mathrm{meV}$ and $d=60 \AA$. The labels S2D and Q2D have the same meaning as in Fig. 2.

where $\mathcal{M}^{a}$ is the average macroscopic pseudospin polarization, which becomes equal to $\left\langle\hat{S}_{\text {tot }}^{a}\right\rangle$ in the limit $I \rightarrow 0$. In the first line of Eq. (7) we have added a Slonczewski [18] pseudospin-transfer term proportional to the tunnel current $I$, which is injected in one layer and extracted from the other. As in the ferromagnetic case, it is the reaction counterpart of the torque which acts on the transport quasiparticles to enable their transfer between layers upon moving through the sample, and must be present because of the nearly exact conservation of pseudospin by interactions. In the second line of Eq. (7) we have added a Gilbert-like damping term $\propto \partial_{t} \mathcal{M}_{x}$ (the anisotropy of the Gilbert damping in the present problem derives from the strongly anisotropic character of the interaction part of the Hamiltonian). These equations [which describe a damped pseudospin precession of frequency $\omega_{\perp}$ and damping rate $\Gamma_{\perp}$ about the steady state values $\mathcal{M}^{y}(t \rightarrow$ $\infty)=I /\left(e \Delta_{\mathrm{SAS}}\right), \mathcal{M}^{x}(t \rightarrow \infty)=0$ ] are similar to those which describe spin-transfer torque oscillators [10] in ferromagnets and suggest that similar, and possibly more flexible, devices could be realized in semiconductor bilayers. We anticipate that the pseudospin resonance will have negative rather than positive dispersion, because of the $q$ dependence of $V_{-}(q)$. The roles of this property, and the fact that the single-particle and collective excitation frequencies are not widely separated, are difficult to fully anticipate. Nevertheless, this work suggests that experimental studies of nonlinear transport in bilayers have great potential.

We thank S. Luin, V. Pellegrini, and L. Sorba for helpful discussions. M.P. acknowledges the hospitality of the Department of Physics and Astronomy of the University of Missouri-Columbia. A.H.M. was supported by the
Welch Foundation, the ARO, and SWAN-NRI. G. V. was supported by NSF Grant No. DMR-031368. B. T. is supported by TUBITAK (No. 106T052) and TUBA.

*m.polini@sns.it

[1] A. H. MacDonald, P. M. Platzman, and G. S. Boebinger, Phys. Rev. Lett. 65, 775 (1990).

[2] J.P. Eisenstein and A.H. MacDonald, Nature (London) 432, 691 (2004).

[3] S. Conti and G. Senatore, Europhys. Lett. 36, 695 (1996); L. Zheng, M. W. Ortalano, and S. Das Sarma, Phys. Rev. B 55, 4506 (1997).

[4] R. Decca et al., Phys. Rev. Lett. 72, 1506 (1994); A. S. Plaut et al., Phys. Rev. B 55, 9282 (1997); D. S. Kainth et al., J. Phys. Condens. Matter 12, 439 (2000); Phys. Rev. B 59, 2095 (1999); S. Holland et al., Phys. Rev. B 66, 073305 (2002).

[5] S. Das Sarma and A. Madhukar, Phys. Rev. B 23, 805 (1981); G.E. Santoro and G.F. Giuliani, ibid. 37, 937 (1988).

[6] S. Das Sarma and E. H. Hwang, Phys. Rev. Lett. 81, 4216 (1998). Notice that within this RPA calculation the frequency of transverse plasmon at $q=0$ is a strictly linear function of the interlayer separation $d$.

[7] P. G. Bolcatto and C. R. Proetto, Phys. Rev. Lett. 85, 1734 (2000).

[8] See, e.g., V. Korenman and R. E. Prange, Phys. Rev. B 6, 2769 (1972); J. Sinova et al., ibid. 69, 085209 (2004); Y. Tserkovnyak, A. Brataas, G.E.W. Bauer, and B. I. Halperin, Rev. Mod. Phys. 77, 1375 (2005), and other related works cited in these papers.

[9] E. M. Hankiewicz, G. Vignale, and Y. Tserkovnyak, Phys. Rev. B 75, 174434 (2007).

[10] S.I. Kiselev et al., Nature (London) 425, 380 (2003); W.H. Rippard et al., Phys. Rev. Lett. 92, 027201 (2004); A. A. Tulapurkar et al., Nature (London) 438, 339 (2005).

[11] J. P. Eisenstein, L. N. Pfeiffer, and K. W. West, Appl. Phys. Lett. 57, 2324 (1990).

[12] G.F. Giuliani and G. Vignale, Quantum Theory of the Electron Liquid (Cambridge University Press, Cambridge, 2005).

[13] R. Nifosì, S. Conti, and M. P. Tosi, Phys. Rev. B 58, 12758 (1998); I. D'Amico and G. Vignale, ibid. 62, 4853 (2000); Z. Qian and G. Vignale, Phys. Rev. Lett. 88, 056404 (2002).

[14] The expression for the resonance position at second order is cumbersome and will be presented elsewhere.

[15] A contribution $-\pi\left(\mathcal{M}_{0}^{z}\right)^{3} \delta\left(\omega-\Delta_{\text {SAS }}\right) / 2$ has been omitted in Eq. (6), since it vanishes at the resonant frequency.

[16] The expression for $\gamma$ is cumbersome and will be presented elsewhere.

[17] See, e.g., R. Côté, L. Brey, and A.H. MacDonald, Phys. Rev. B 46, 10239 (1992).

[18] J. A. Slonczewski, J. Magn. Magn. Mater. 159, L1 (1996). 